\title{
Low-loss, single-mode photonic wires and ring resonators in Silicon-on-Insulator
}

\author{
Pieter Dumon ${ }^{1}$, Wim Bogaerts ${ }^{1}$, Joris Van Campenhout ${ }^{1}$, Vincent Wiaux ${ }^{2}$, \\ Johan Wouters ${ }^{2}$, Stephan Beckx ${ }^{2}$, Roel Baets ${ }^{1}$ \\ ${ }^{1}$ Ghent University - IMEC, Dept. of Information Technology, Sint-Pietersnieuwstraat 41 \\ 9000 Gent, BELGIUM, pieter.dumon@intec.UGent.be \\ ${ }^{2}$ IMEC vzw, Silicon Process Technology Division, Kapeldreef 75, 3001 Leuven, BELGIUM
}

Abstract We demonstrate single-mode photonic wires in Silicon-on-insulator with propagation losses as low as $2.4 \mathrm{~dB} / \mathrm{cm}$. We also constructed compact racetrack and resonators in these waveguides with a Q larger than 3000 .

\section{Introduction}

One of the main requirements for the further integration of photonics is a low-loss waveguide that allows for short bends. This can be done using traditional index-confined waveguides with a high refractive index contrast. In these so-called photonic wires, bend radii as small as $2 \mu \mathrm{m}$ are possible. However, in such narrow waveguides, the principal loss mechanism is scattering at sidewall roughness[1]. Therefore, very accurate fabrication processes are needed. By thermally oxidising the top silicon layer, the sidewalls can be smoothened, resulting in lower losses [2][3]. However, oxidation also reduces the core area, which can result in a less confined mode, which in its turn limits the bend radius.

We have fabricated photonic wires using deep UV lithography[4]. To keep sidewall roughness within bounds, we did not etch the buried oxide cladding was not etched. This structure is illustrated in figure 1 . No additional roughness reduction like thermal oxidation was applied.

\section{Straight waveguides}

To characterise the waveguide losses we measured the transmission spectrum of a cavity formed by 2 cleaved facets in a $3 \mu \mathrm{m}$ wide ridge waveguide. In the middle of the cavity, the waveguide was tapered down to a photonic wire using an adiabatic taper. We measured various cavities with different lengths of photonic wires. From the Fabry-Pérot transmission spectrum we could then extract the total cavity loss. When plotted on a logarithmic scale as a function of length, the propagation loss of the photonic wires can be extracted from the slope of the fitted straight line.
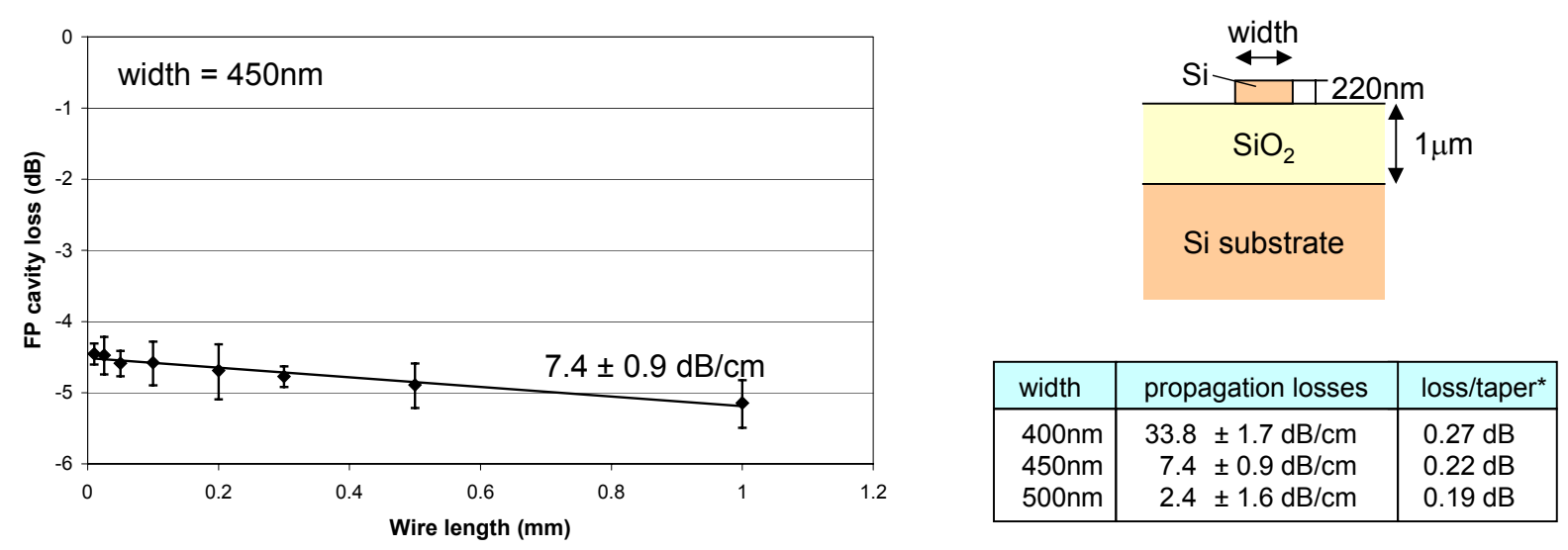

Figure 1: Total Fabry-Pérot cavity loss (dB) for cavities with 450nm photonic wires with different lengths. The wire loss can be extracted from the slope of the fitted line. The sum of facet reflection and loss of the tapers can be calculated from the intercept. From this, we estimate an upper boundary for the loss per taper. 
We measured the propagation losses for wires of various length, and we see that the losses decrease exponentially for broader wires. This is in agreement with sidewall roughness as the primary loss mechanism. For a 500nm wire, which is still a single-mode waveguide, we have measured a propagation loss of $2.4 \mathrm{~dB} / \mathrm{cm}$. For this waveguide, $76 \%$ of the light is confined within the core, making it suitable for short bends. We can also make an estimate of the paer losses, by replacing the facet reflection by its theoretical value as an upper boundary. This gives an upper boundary of $0.19 \mathrm{~dB}$ for the loss of a $200 \mu \mathrm{m}$ long taper from $3 \mu \mathrm{m}$ width down to a $500 \mathrm{~nm}$ wide wire.

\section{Resonators}

Resonators can provide building blocks for a large number of functional components on a photonic IC. In photonic wires, ring resonators are the easiest to implement. Figure 2 shows the transmission spectrum of a ring resonator with a straight coupling section (a so-called racetrack resonator). The resonator has a quality factor Q of over 3000, a free spectral range of around $14 \mathrm{~nm}$ and a finesse of 27.

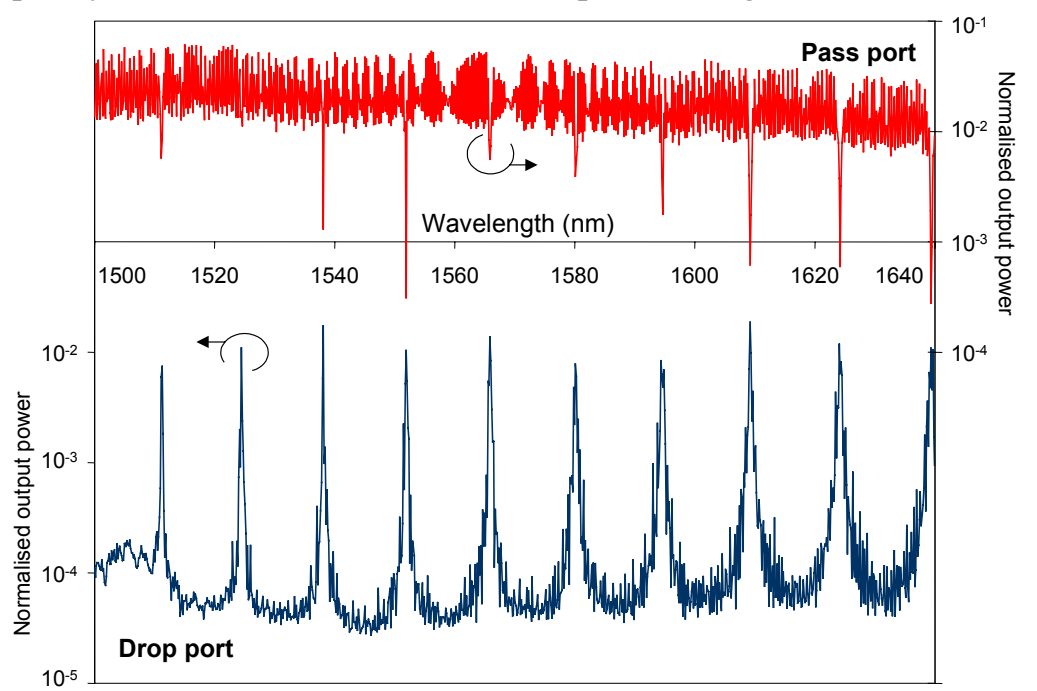

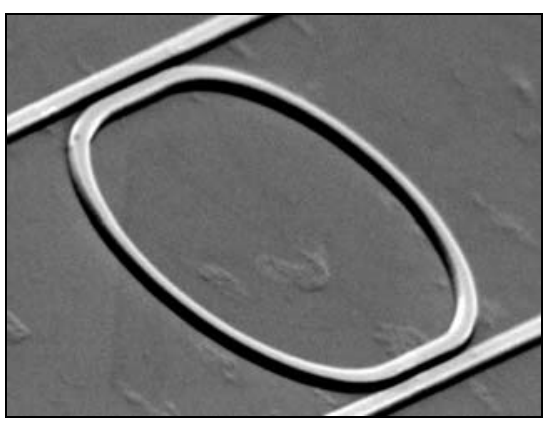

Wire width $=500 \mathrm{~nm}$

Gap width $=230 \mathrm{~nm}$

Figure 2: Transmission spectrum of a racetrack resonator with a $Q=3086$. Note that the scales for pass and drop powers are shifted. The bend radius and the straight section length are $3 \mu \mathrm{m}$. The large fluctuations in the pass port power is due to the Fabry-Pérot cavity formed by the cleaved facets.

\section{Conclusion}

We have demonstrated single-mode photonic wires in SOI fabricated with deep UV lithography. The measurements show propagation losses down to $2.4 \mathrm{~dB} / \mathrm{cm}$ for a $500 \mathrm{~nm}$ waveguide. We also demonstrated a racetrack resonator with a $\mathrm{Q}>3000$.

\section{Acknowledgements}

Part of this work was done in the context of the IST-PICCO project supported by the European Union. Part of this work was carried out in the context of the Belgian IAP PHOTON network Wim Bogaerts and Pieter Dumon thank the Flemish Institute for the industrial advancement of scientific and technological Research (IWT) for a specialisation grant.

Joris Van Campenhout was supported by the Flemish Fund for Scientific Research (FWO - Vlaanderen) through a doctoral fellowship.

\section{References}

[1] W. Bogaerts et al, Opt. Lett. 28(9), p. 689

[2] K. K. Lee et al. Opt. Lett. 26(23), p.1888

[3] T. Tsuchizawa et al., LEOS Annual Meeting 2002, Glasgow, UK, p.287

[4] W. Bogaerts et al, IEEE. J. Sel. Top. Quant. Electron. 8(4), p.928 